\title{
Fröhlich Systems in Cellular Physiology
}

\section{Šrobár F.}

Institute of Photonics and Electronics, Academy of Sciences of the Czech Republic, Prague, Czech Republic

Received December 20, 2011; Accepted April 11, 2012.

Key words: Fröhlich systems - Bioelectromagnetic phenomena - Microtubules Causal diagrams

Abstract: Electromagnetic fields are usually absent in the picture of processes taking place in living cells which is dominated by biochemistry, molecular genetics and microscopic morphology. Yet experimental and theoretical studies suggest that this omission is not justified. At the end of 1960's H. Fröhlich elaborated a semi-phenomenological model of polar oscillating units that are metabolically driven, exchange energy with the cell's internal heat reservoir, and store part of the energy in excited vibrational modes in such way, that mode with the lowest frequency becomes highly excited, while the higher-order modes remain near thermal equilibrium. This affords energy-hungry chemical reactions to take place while the rest of the cell is not exposed to heat stress. At present, part of the cytoskeleton - microtubules - are deemed to fulfil the role of oscillating units. The paper provides an introduction to the Fröhlich ideas for readers with background in medicine and biology in that it avoids mathematical formulas and relies on figures to convey information about the basic properties of the model. The essential features of the Fröhlich model - most notably the energy condensation - are demonstrated on ensemble encompassing three coupled vibration modes that can be exactly described using original diagrammatic method.

This study was supported by grant No. P102/11/0649 of the Czech Science Foundation GA CR.

Mailing Address: Ing. Fedor Šrobár, DSc., Institute of Photonics and Electronics, Academy of Sciences of the Czech Republic, Chaberská 57, 18251 Prague 8, Czech Republic; Phone: +420 266773 473; Fax: +420 284680 222; e-mail: srobar@ufe.cz 


\section{Introduction}

There is a remarkable disproportion between the abundant current knowledge of the cell's biochemistry and genomic equipment on one hand and the much lesser information available about the cell as a physical universe on the other. An early attempt to rectify this situation was undertaken in late sixties of the previous century by Anglo-German physicist Herbert Fröhlich (1968a, b). He was intrigued by the presence of cellular macromolecules with significant electric dipoles which are bound to emit electromagnetic fields, being excited by the cell's metabolic energy. Individual molecular oscillators interact with each other, giving rise to collective motion which comes in the form of discrete modes of vibration. Analyzing a mathematical model of such situation, Fröhlich found that, under suitable conditions, the supplied energy is deposited preferentially in the mode with lowest frequency. This mode then becomes excited far beyond the thermal-equilibrium level, even though the rest of the system remains close to equilibrium. This could have far-reaching consequences, e.g. for the kinetics of chemical reactions inside the cell, causing departure from the usual Arrhenius scheme and transition-state concept.

Fröhlich's suggestions generated a flurry of both theoretical and experimental work in the 1970's and 1980's; later on this activity receded because empirical manifestations proved very elusive. Experimental study of cellular electromagnetic fields is a formidable task indeed: the emitted power is in the range of femto-Watts $\left(10^{-15} \mathrm{~W}\right)$, the usual recourse to cryogenic temperatures to avoid thermal noise cannot be taken because the cell's normal activity must be preserved. In the 1990's, the only two groups that continued experimental study in this direction were those of Prague (Jelínek et al., 1999) and Berlin (Hölzel and Lamprecht, 1995). Using ultrasensitive measuring equipment with perfect electromagnetic shielding, together with sophisticated data processing procedures that afford retrieval of feeble relevant signals from noisy background, Pokorný and his group localized cell-emitted radiation near $8 \mathrm{MHz}$ on synchronized cultures of Saccharomyces cerevisiae (Pokorný et al., 2001). In recent years there came a revival of interest in Fröhlich theory, with energy transfer in photosynthetic complexes being an additional field of its application (Engel et al., 2007). Also, the much discussed and publicized Penrose-Hameroff orchestrated objective reduction hypothesis of human consciousness (Hameroff and Penrose, 1996) makes reference to this theory.

The aim of this paper is to explain basic features of the Fröhlich theory to readers with background in medicine and biology. The author tried not to use mathematic formulas in the text; the main vehicles of explanation are the figures depicting important properties of the model. A brief glossary of selected relevant notions at the end of the paper is also intended to assist the reader not versed in physics.

Systems of two or three coupled oscillators were shown to represent plausibly general properties of the Fröhlich systems (Šrobár and Pokorný, 1996, 1999; Šrobár, 2005, 2009); such approach is particularly useful in an introductory text. As to the oscillating polar entities, Fröhlich envisaged protein molecules embedded in cellular 
membranes. Now, in eukaryotic cells, it is mainly the constituents of cytoskeleton the microtubules - that are believed to fulfil this role (Pokorný, 2004). Mitochondria enter the picture as energy providers and source of strong static electric fields that support nonlinear behaviour which is essential to the unfolding of all features of the Fröhlich model. Electric fields of mitochondria, as well as those created by charges residing on molecules and various polarized interfaces in the cell' interior, cause the water to be organized. This, among other effects, lowers the viscous damping of microtubule vibrations (Pokorný, 2004; Pollack et al., 2006; Zheng et al., 2006).

\section{The Fröhlich model}

An ensemble of $N$ oscillating and mutually interacting polar units, receiving energy from a source and in contact with a heat bath (Figure 1) is equivalent to ensemble of $N$ collective modes of vibration with discrete frequencies $v_{1}<v_{2}<\ldots<v_{N}$ covering certain frequency band. As we are dealing with molecules, excitation of the individual modes is expressed in the number of energy quanta or occupation numbers $n_{\mathrm{i}}$. As indicated in the figure, the interaction with the heat bath can be either linear - exchange of energy quantum between the mode and the bath - so called $\Phi$-process, proportional to a numerical coefficient $\Phi$, or it can be nonlinear (X-process, proportional to coefficient $X$, the capital variety of Greek $\chi$ ), whereby two modes exchange a quantum with participation of the bath. (Stricto sensu, interaction between elastic and polarization fields leads to dependence of the coefficients $\Phi$ and $X$ on excitation. To keep the explanation simple, we neglect this nonessential dependence.) Fröhlich proposed equations describing balance of the occupation numbers; the total rate of change (per second) of the occupation number $n_{\mathrm{i}}$ equals the difference between creation of quanta by pumping $s$ provided by metabolic sources minus the net loss of quanta due to linear and nonlinear interaction processes.

There are $N$ equations of this type $(i=1,2, \ldots, N)$, they are mutually coupled and nonlinear.

Interaction:

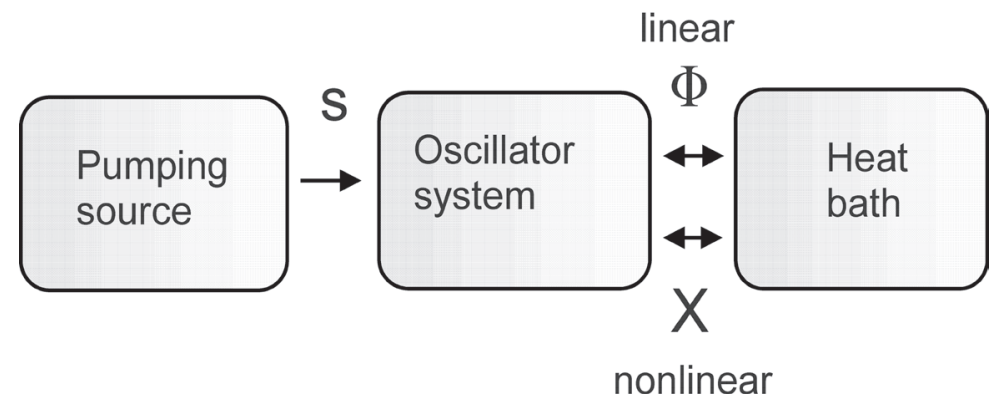

Figure 1 - Conceptual elements of the Fröhlich model. 
We elaborated a diagrammatical method affording numerical evaluation of such equation systems (Šrobár and Pokorný, 1996, 1999); the dependences shown below were obtained using this procedure.

\section{The phenomenon of energy condensation}

We have shown (Šrobár and Pokorný, 1999; Šrobár, 2005, 2009) that ensembles counting just two or three coupled oscillators (the behaviour of which can be described exactly) evince the hallmark properties of the Fröhlich systems, such as the energy condensation. Figure 2 details the scheme of Figure 1 for the case of three oscillators. The remaining figures (Figures 3-7) present examples of such study for $N=3$, in the form of dependences of modal occupancy numbers $n_{i}(i=1,2$, and 3 ) on the pumping rate $s$ for the steady-state condition when the occupation numbers do not vary in time. In all figures, value of the linear-exchange coefficient $\Phi$ was kept fixed at $0.01 \mathrm{~s}^{-1}$; three values of the nonlinear-exchange coefficient were used, $X=0.001,1 \times 10^{-6}$ and $0 \mathrm{~s}^{-1}$, representing strong, medium, and nonexistent nonlinear coupling of the ensemble with the heat bath.

Figures 3 and 4 refer to the 10-100 MHz radio-frequency domain, close to frequency of the yeast cells electromagnetic emission observed by our group (Jelínek et al., 1999). The dependences for strong and feeble nonlinearity $X$ coalesce in this case (Figure 3) and exhibit clearly the condensation of incoming energy in the lowest-order mode 1: whereas $n_{2}$ and $n_{3}$ do not virtually change with increasing pumping level $s$, remaining close to their thermal equilibrium values $n_{20}$ and $n_{30}$, the population of the fundamental mode increases for $s$ surpassing certain level (approximately $3 \times 10^{-3} \mathrm{~s}^{-1}$ in this case). The total absence of nonlinearity $(X=0$, Figure 4) abolishes the condensation phenomenon: all three modes now benefit from the increasing energy influx in equal manner.

Situation in the millimetre-wavelength region of the electromagnetic spectrum is shown in Figures 5-7. The circumstances now are changed due to difference in the ratio of photon energy quantum hv (h being the Planck constant) and energy of the thermal molecular motion $\mathrm{kT}$ ( $\mathrm{k}$ is the Boltzmann constant, $T$ is absolute temperature): for $v=30 \mathrm{MHz}$ this ratio is approximately $5 \times 10^{-6}$, whereas for $v=0.3 \mathrm{THz}$ it is 0.05 . As a consequence, the population levels are now shifted about four orders of magnitude lower (see difference in the vertical scales). As in the radio-frequency case, total absence of nonlinearity $(X=0$, Figure 7 ) means no energy condensation - populations of all three modes increase with enhanced pumping. Unlike the lower frequency case, however, the strong and medium nonlinearity instances yield different pictures: for $X=0.001 \mathrm{~s}^{-1}$ (Figure 5) there is a full-fledged condensation of energy in the lowest-order mode, whereas for $X=10^{-6} \mathrm{~s}^{-1}$ (Figure 6) one can see instance of an imperfect condensation, with the populations of higher-order modes, $n_{2}$ and $n_{3}$ also benefiting to a certain degree from the energy pumped into the system while the fundamental mode still has privileged access to it. 


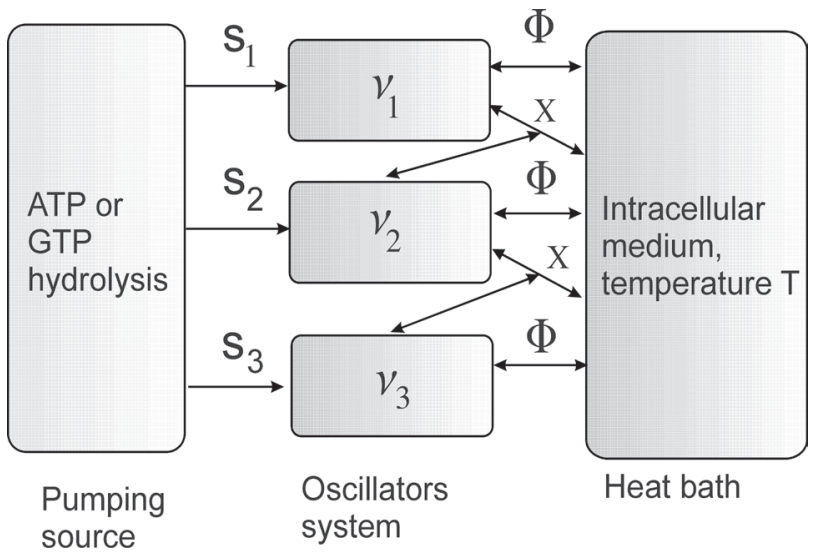

Figure 2 - Interactions in a three-mode Fröhlich system. Meaning of the symbols is explained in the main text. To keep the picture uncluttered, only some of the $X$-interactions are indicated, namely those between modes neighbouring in the frequency spectrum $v_{1}<v_{2}<v_{3}$.

Figure 3 - Dependences of occupation numbers ni of the three modes on the pumping rate $s$ for modal frequencies in the tens of $\mathrm{MHz}$ region $\left(v_{1}=10 \mathrm{MHz}, v_{2}=30 \mathrm{MHz}\right.$, $v_{3}=100 \mathrm{MHz}$.

The case of strong nonlinearity, $X=0.001 \mathrm{~s}^{-1}$. The curves for the medium nonlinearity, $X=1 \times 10^{-6} s^{-1}$ coalesce with those for the strong one. Value of the linear interaction coefficient is $\Phi=0.01 \mathrm{~s}^{-1}$. One can observe condensation of energy in the lowest-order mode indexed as 1.
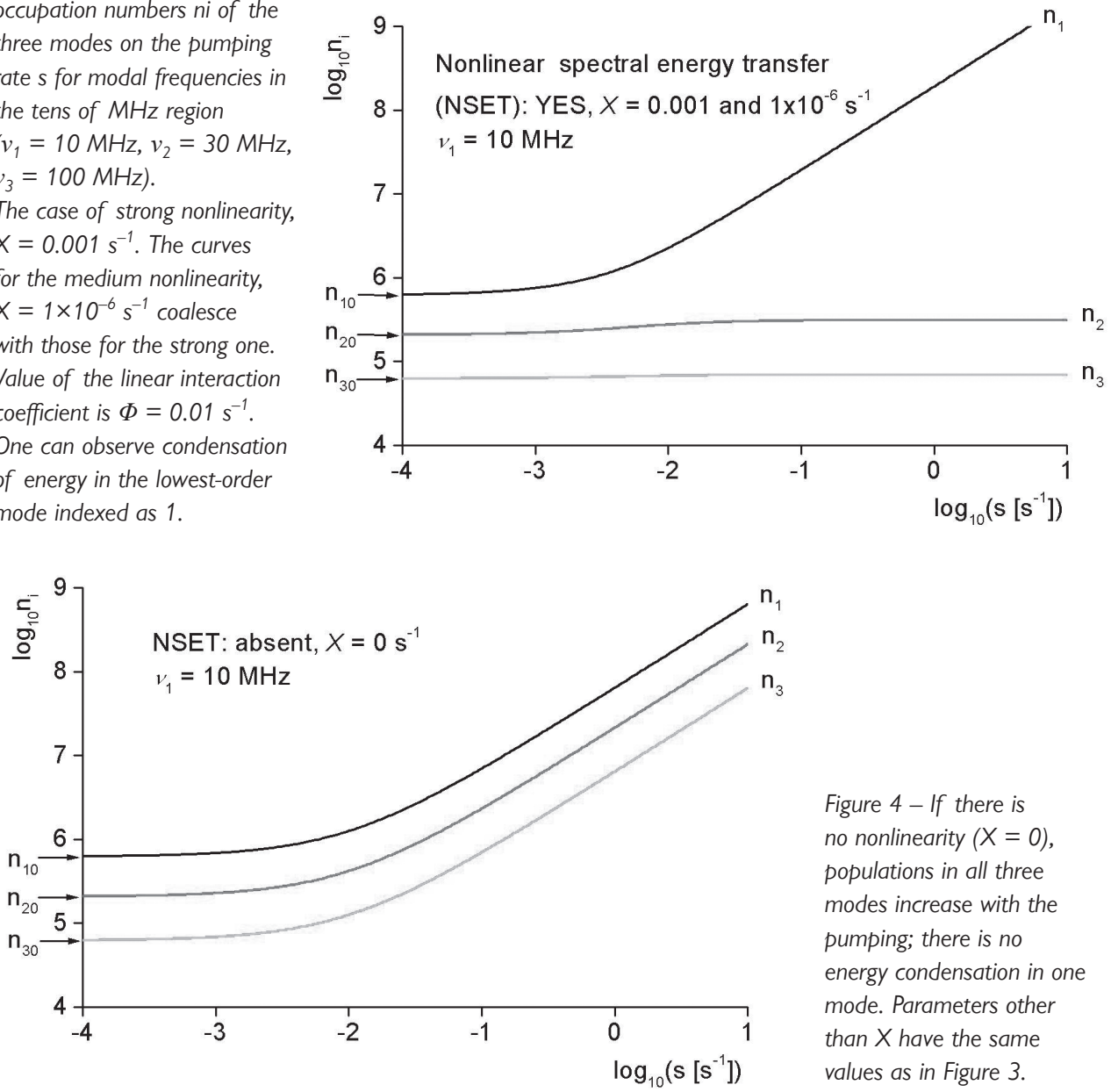

Figure $4-$ If there is no nonlinearity $(X=0)$, populations in all three modes increase with the pumping; there is no energy condensation in one mode. Parameters other than $X$ have the same values as in Figure 3.

Fröhlich Systems in Cellular Physiology 


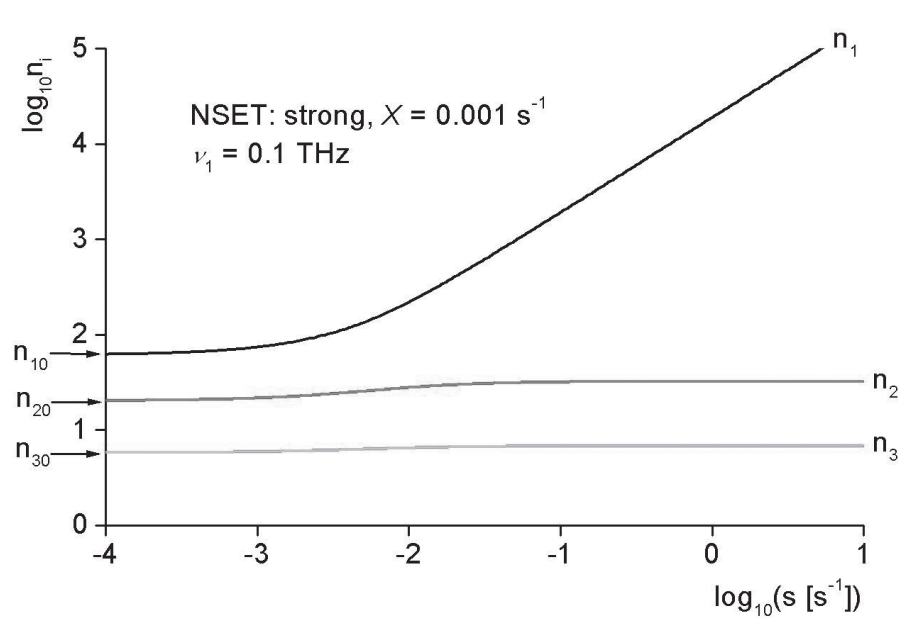

Figure 5 - Occupation numbers versus pumping rate dependences for modal frequencies in the millimetrewavelength region $\left(v_{1}=\right.$ $0.1 \mathrm{THz}, v_{2}=0.3 \mathrm{THz}$ $v_{3}=1 \mathrm{THz}$ ). Strong nonlinearity, $X=0.001 \mathrm{~s}^{-1}$. Linear interaction coefficient $\Phi=0.01 \mathrm{~s}^{-1}$. Condensation of energy in the lowest-order mode.

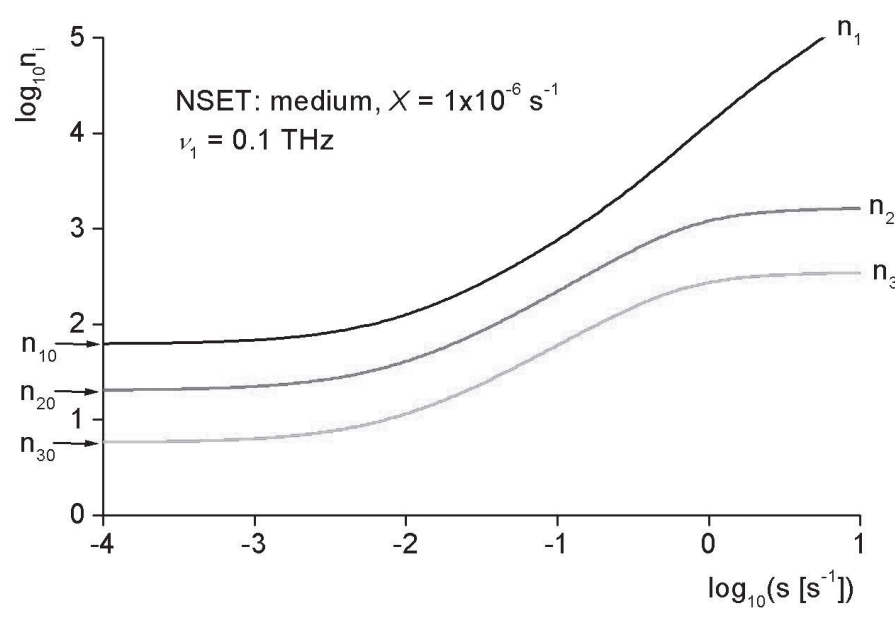

Figure 6 - The case of medium nonlinearity, $X=1 \times 10^{-6} \mathrm{~s}^{-1}$, other parameter values as in Figure 5. While most of the incoming energy is deposited in the first-order mode, the populations in the higher-order modes are also increased to a certain degree.

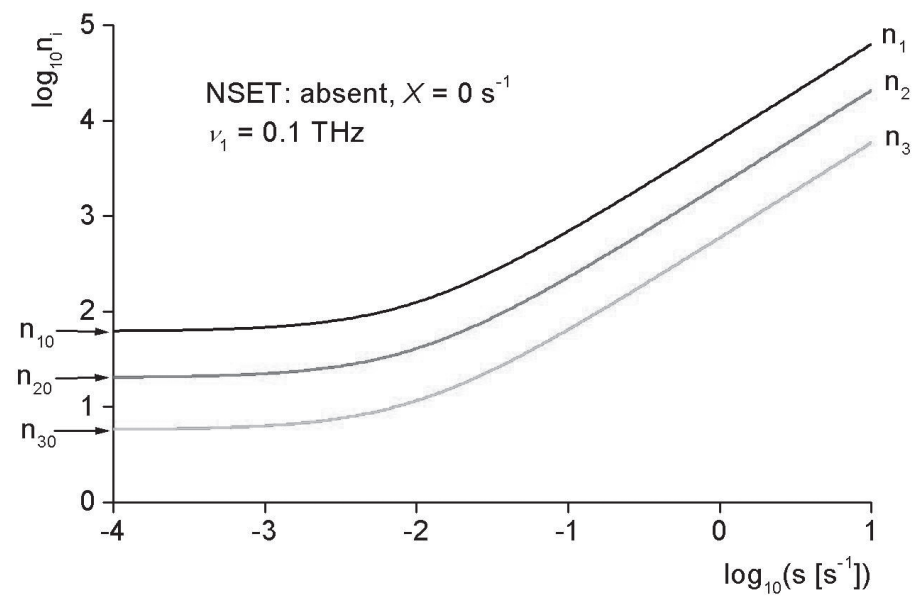

Figure 7 - The case of vanishing nonlinearity, $X=0$, other parameter values as in Figure 5. Populations in all three modes increase with the pumping; there is no energy condensation in one mode.

Šrobár F. 


\section{Discussion}

From the point of view of cellular physiology, the most relevant property of the Fröhlich model is the possibility to concentrate great number of energy quanta in one vibrational mode, significantly above the thermal equilibrium level, whereas the system as a whole remains close to equilibrium with the heat bath. The excited mode can be a party to chemical reaction that would not take place near equilibrium while the bulk of the cell's interior is not exposed to energetic stress.

Another property associated with the Fröhlich systems is coherence - a correlation or a kind of commonality between individual oscillations. Like energy condensation, the coherence can also have profound impact on cell's energetics. For instance, a resonant energy transfer between molecules forming reaction centres of photosynthetic bacteria is much more efficient than a stochastic one (Vos et al., 1994; Engel et al., 2007). In this quantum-mechanical process the light-induced energy excitation is transferred in a coherent wavelike manner from the donor to the acceptor components of the photosynthetic complex. The high efficiency of this transfer is afforded by the surprisingly large coherence time of $0.3 \mathrm{ps}=3 \times 10^{-13} \mathrm{~s}$ (Scholes, 2010). It should be noted that energy condensation and coherence are independent aspects of the model and do not come necessarily together.

The pumping sources in Figures 1 and 2 stand, in eukaryotic cells, for ATP or GTP-producing mitochondria. There is a proton gradient across the mitochondrial membrane whose electrochemical potential powers the ATP synthase. It also creates a zone of strong electrostatic field (Tyner et al., 2007, report field intensity of 3.5 $\mathrm{MV} \mathrm{m}^{-1}$ ), extending several micrometers from the mitochondrion. Given the fact that mitochondria are aligned in the vicinity of microtubules, the latter are immersed in this field. Both the tubulin heterodimers and microtubules possess strong electric dipoles that interact with the field. This promotes nonlinear properties represented in the Fröhlich model (and in Figures 3-7) by the coefficient $X$. Such influence is mediated by deformation (loss of symmetry) of the potential profile in which dipoles are embedded which leads to nonlinear terms in field dependence of polarization - a phenomenon encountered also in nonlinear optics (Nayar and Winter, 1990; Boyd, 2008). Strong electric fields also lead to organization of water which reduces damping of microtubule oscillations. Insufficient awareness of the nonlinear aspects of the Fröhlich model leads to denial of its applicability under physiological conditions (McKemmish et al., 2009; Reimers et al., 2009).

The coupling with the heat bath, expressed by the coefficients $\Phi$ and $X$, represents a damping of the oscillator system. An increase of these coefficients results in shifting of the curves in Figures 3-7 to the right: higher pumping levels are required to produce the upward bending of the modal population curves. If this leaking of energy to surroundings is too high, there is no energy condensation at physiologically admissible pumping rates. Such situation can occur e.g. if the oscillating units are phosphorylated - a condition which enhances losses to the surroundings (Pokorný and Fiala, 1992). 


\section{Conclusion}

Fröhlich's model describes behaviour of a thermodynamically open system of collective vibrational modes receiving energy from metabolic sources and giving it off to the heat bath. The energy dissipation processes can be linear, involving simple exchange with the states of surroundings, or nonlinear, with interactions encompassing two system and one heat bath states. A marked feature of this model is the possibility of energy condensation in the lowest-order vibrational mode. In the context of cellular biochemistry, this affords engagement in reactions requiring highly excited states, while most of the system remains close to thermal equilibrium, causing no stress to the cell. Another aspect of the Fröhlich's model, the coherence, apparently plays an important role in intermolecular energy transfer.

\section{Appendix: explanation of some physical terms}

Coherence. Even when restricted to the realm of physics, this term has a variety of nuances. The most straightforward definition refers to two harmonic (like sine or cosine) waves of equal frequency: they are coherent if their relative phase remains constant. In that case they can interfere if they meet. The state of coherence (phase memory) is gradually eroded by stochastic influences (fluctuations) of the milieu in which the wave propagates - hence one speaks of coherence length or coherence time during which the coherence is, to some degree, preserved. Quantum coherence is a phenomenon without close classical analogue, it refers to phase of wave functions describing states of particles in the microworld. It is relevant in processes of resonant energy transport between molecules.

Energy condensation. $\mathrm{H}$. Fröhlich borrowed this appellation from quantum statistics (Bose-Einstein condensation); in the present context it designates the situation in which the population of the lowest-order (and lowest-energy) vibrational mode significantly exceeds its thermal-equilibrium level.

Energy quantum. Fröhlich model, in its original form, is semi-phenomenological, nonetheless, it uses the quantum-mechanical notion of energy quanta: the degree of excitation of modes is expressed in terms of number of energy quanta $n_{\mathrm{i}}$ residing in the $i$-th mode. This energy is proportional to the modal frequency $v_{\mathrm{i}}$.

Mode of vibration. Wave phenomena are described by partial differential equations that provide a continuum of solutions. If one places additional constraints (like boundary conditions dictated by given physical situation), only a discrete subset of solutions is admissible. They can be characterized by sets of integers (in our case just one integer, $i=1,2, \ldots, N)$ and are called the modes. As an example, in a string fixed at the ends, only vibrations with an integer number of half-wavelengths per string length are allowed.

Linear and nonlinear coupling. In the context of the Fröhlich model, linear processes involve exchange of energy between one vibration mode and the heat bath creation or annihilation of energy quantum pertaining to this mode. Nonlinear processes encompass energy exchange between two modes with participation of the 
heat bath. In the rate equations for occupation numbers $n_{i}$ there appear terms like $n_{\mathrm{i}} n_{\mathrm{j}}$, which means mathematical nonlinearity. It must be kept in mind that the Fröhlich system is pumped and therefore off equilibrium. Similar processes of spectral energy redistribution occur in pumped nonlinear optical materials.

\section{References}

Boyd, R. W. (2008) Nonlinear Optics. Academic Press, London.

Engel, G. S., Calhoun, T. R., Read, E. L., Ahn, T. K., Mancal, T., Cheng, Y. C., Blankenship, R. E., Fleming, G. R. (2007) Evidence for wavelike energy transfer through quantum coherence in photosynthetic systems. Nature 446, 782-786.

Fröhlich, H. (1968a) Bose condensation of strongly excited longitudinal electric modes. Phys. Lett. A 26, 402-403.

Fröhlich, H. (1968b) Long-range coherence and energy storage in biological systems. J. Quantum Chem. II, 641-649.

Hameroff, S. R., Penrose, R. (1996) Conscious events as orchestrated space-time selections. J. Conscious. Stud. 3, 36-53.

Hölzel, R., Lamprecht, I. (1995) Optimizing an electronic detection system for radiofrequency oscillations in biological cells. Neural Network World 5, 763-774.

Jelínek, F., Pokorný, J., Šaroch, J., Trkal, V., Hašek, J., Palán, B. (1999) Microelectronic sensors for measurement of electromagnetic fields of living cells and experimental results. Bioelectrochem. Bioenerg. 48, 261-266.

McKemmish, L. K., Reimers, J. R., McKenzie, R. H., Mark, A. E., Hush, N. S. (2009) Penrose-Hameroff orchestrated objective-reduction proposal for human consciousness is not biologically feasible. Phys. Rev. E Stat. Nonlin. Soft Matter Phys. 80, 021912-1-021912-6.

Nayar, B. K., Winter, C. S. (1990) Organic second-order non-linear optical materials and devices. Opt. Quantum Electron. 22, 297-318.

Pokorný, J. (2004) Excitation of vibrations in microtubules in living cells. Bioelectrochemistry $\mathbf{6 3}$, 321-326.

Pokorný, J., Fiala, J. (1992) Heat bath coupling effects in coherent vibration systems. Europhys. Lett. 19, 729-734.

Pokorný, J., Hašek, J., Jelínek, F., Šaroch, J., Palán, B. (2001) Electromagnetic activity of yeast cells in the M phase. Electro Magnetobiol. 20, 371-396.

Pollack, D., Cameron, I., Wheatley, D. (2006) Water and the Cell. Springer, Dordrecht.

Reimers, J. R., McKemmish, L. K., McKenzie, R. H., Mark, A. E., Hush, N. S. (2009) Weak, strong, and coherent regimes of Fröhlich condensation and their applications to terahertz medicine and quantum consciousness. Proc. Natl. Acad. Sci. USA 106, 4219-4224.

Scholes, G. D. (2010) Green quantum computers. Nat. Phys. 6, 402-403.

Šrobár, F. (2005) Fröhlich system with modulated access to pumping source. Electromagn. Biol. Med. 24, $265-272$.

Šrobár, F. (2009) Occupation-dependent access to metabolic energy in Fröhlich systems. Electromagn. Biol. Med. 28, 194-200.

Šrobár, F., Pokorný, J. (1996) Topology of mutual relationships implicit in the Fröhlich model. Bioelectrochem. Bioenerg. 41, 31-33.

Šrobár, F., Pokorný, J. (1999) Causal structure of the Fröhlich model of cellular electromagnetic activity. Electro Magnetobiol. 18, 257-268. 
104) Prague Medical Report / Vol. 113 (2012) No. 2, p. 95-104

Tyner, K. M., Kopelman, R., Philbert, M. A. (2007) "Nanosized voltmeter" enables cellular-wide electric field mapping. Biophys. J. 93, 1163-1173.

Vos, M. H., Jones, M. R., Hunter, C. N., Breton, J., Martin, J. L. (1994) Coherent nuclear dynamics at room temperature in bacterial reaction centers. Proc. Natl. Acad. Sci. USA 91, 12701-12705.

Zheng, J., Chin, W., Khijniak, E., Khijniak, E. Jr., Pollack, G. H. (2006) Surfaces and interfacial water: evidence that hydrophilic surfaces have long-range impact. Adv. Colloid Interface Sci. 127, 19-27. 